\title{
Metallic Hyperdensity Sign on Noncontrast CT Immediately after Mechanical Thrombectomy Predicts Parenchymal Hemorrhage in Patients with Acute Large-Artery Occlusion
}

\author{
(D). Xu, (D) Y. Zhou, DR. Zhang, (D) Z. Chen, (D). Zhong, (D) X. Gong, (D). Ding, and (D). Lou
}

\begin{abstract}
BACKGROUND AND PURPOSE: Parenchymal hemorrhage is a severe complication following mechanical recanalization in patients with acute ischemic stroke with large-vessel occlusion. This study aimed to assess whether the metallic hyperdensity sign on noncontrast CT performed immediately after mechanical thrombectomy can predict parenchymal hemorrhage at 24 hours.
\end{abstract}

MATERIALS AND METHODS: We included consecutive patients with acute ischemic stroke with large-vessel occlusion who underwent noncontrast CT immediately after mechanical thrombectomy between January 2014 and September 2018. The metallic hyperdensity sign was defined as a nonpetechial intracerebral hyperdense lesion (diameter, $\geq 1 \mathrm{~cm}$ ) in the basal ganglia and a maximum CT density of $>90 \mathrm{HU}$. The sensitivity, specificity, and positive and negative predictive values of the metallic hyperdensity sign in predicting parenchymal hemorrhage were calculated.

RESULTS: A total of 198 patients were included. The metallic hyperdensity sign was found in 59 (29.7\%) patients, and 51 (25.7\%) patients had parenchymal hemorrhage at 24 hours. Patients with the metallic hyperdensity sign are more likely to have parenchymal hemorrhage than those without it $(76.3 \%$ versus $4.3 \%, P<.001)$. The sensitivity, specificity, positive predictive value, and negative predictive value of the metallic hyperdensity sign in predicting parenchymal hemorrhage were $88.2 \%, 90.5 \%, 76.3 \%$, and $95.7 \%$, respectively.

CONCLUSIONS: The presence of the metallic hyperdensity sign on noncontrast CT performed immediately after mechanical thrombectomy in patients with large-vessel occlusion could predict the occurrence of parenchymal hemorrhage at 24 hours, which might be helpful in postinterventional management within 24 hours after mechanical thrombectomy.

ABBREVIATIONS: HT = hemorrhagic transformation; $\mathrm{MT}=$ mechanical thrombectomy; $\mathrm{PH}$ = parenchymal hemorrhage

$\mathbf{R}$ eperfusion therapy with mechanical thrombectomy (MT) improves functional outcome in patients with acute ischemic stroke with large-vessel occlusion. ${ }^{1-5}$ However, $>50 \%$ of patients still experience unfavorable outcome. ${ }^{6,7}$ One of the most common reasons is hemorrhagic transformation ( $\mathrm{HT})$, especially parenchymal hemorrhage $(\mathrm{PH})$, which is closely related to deterioration of neurologic symptoms. ${ }^{8,9}$ Early prediction of

Received October 16, 2018; accepted after revision February 7, 2019.

From the Departments of Neurology (C.X., Y.Z., R.Z., Z.C., W.Z, X.G., M.L.) and Radiology (X.D.), the Second Affiliated Hospital of Zhejiang University, School of Medicine, Hangzhou, China.

This study was supported by the National Natural Science Foundation of China (81622017, 81471170), the National Key Research and Development Program of China (2016YFC1301503), the Science Technology Department of Zhejiang Province (2018C04011), and Fundamental Research Funds for the Central Universities (2017XZZX002-09).

Please address correspondence to Min Lou, MD, PhD, Department of Neurology, the Second Affiliated Hospital of Zhejiang University, School of Medicine, 88 Jiefang Rd, Hangzhou, 310009, China; e-mail: Im99@zju.edu.cn

- Indicates open access to non-subscribers at www.ajnr.org

http://dx.doi.org/10.3174/ajnr.A6008
HT, therefore, is important for postinterventional therapy adjustment- that is, whether to start treatment with the glycoprotein IIb/IIIa inhibitor tirofiban to prevent early re-occlusion due to endothelial damage. ${ }^{10}$

In some hospitals, immediate postinterventional non-contrastenhanced CT has been introduced to estimate bleeding complications. Hyperdense areas may frequently be detected on NCCT after intervention. ${ }^{11-14}$ However, such hyperdense areas on NCCT have not been well understood, and different interpretations have been reported in various publications.

For instance, Yoon et $\mathrm{al}^{13}$ demonstrated that intracerebral hyperdense lesions with a maximum Hounsfield unit measurement of $>90$ were highly associated with symptomatic hemorrhage after intra-arterial thrombolysis. On the other hand, Jang et $\mathrm{al}^{14}$ reported that the possibility of HT depended on different morphologic features of intracerebral hyperdense lesions after intra-arterial thrombolysis, and they proposed the concept of metallic hyperdensity lesions, which were closely related to a high proportion of HT after intra-arterial throm- 
Table 1: Predictive value of characteristics of metallic hyperdensity for parenchymal hemorrhage

\begin{tabular}{|c|c|c|c|c|c|c|c|}
\hline & AUC & $95 \% \mathrm{Cl}$ & $\begin{array}{c}P \\
\text { Value }\end{array}$ & Sensitivity & Specificity & $\begin{array}{l}\text { Positive } \\
\text { Predictive } \\
\text { Value }\end{array}$ & $\begin{array}{c}\text { Negative } \\
\text { Predictive } \\
\text { Value }\end{array}$ \\
\hline Presence of metallic hyperdensity sign & 0.894 & $0.835-0.952$ & $<.001$ & 0.882 & 0.905 & 0.763 & 0.957 \\
\hline
\end{tabular}

Note:-AUC indicates area under the curve.

Table 2: Comparison of characteristics between patients with and without the metallic hyperdensity sign

\begin{tabular}{|c|c|c|c|}
\hline Characteristics & $\begin{array}{c}\text { Presence of } \\
\text { Metallic Hyperdensity } \\
\text { Sign }(n=59)\end{array}$ & $\begin{array}{c}\text { Absence of } \\
\text { Metallic Hyperdensity } \\
\text { Sign }(n=139)\end{array}$ & $\begin{array}{c}P \\
\text { Value }\end{array}$ \\
\hline Age (mean) (yr) & $70.5 \pm 10.5$ & $69.4 \pm 12.2$ & .568 \\
\hline Female (No.) (\%) & $26(44.1)$ & $56(40.3)$ & .639 \\
\hline \multicolumn{4}{|l|}{ Comorbid conditions } \\
\hline Hypertension (No.) (\%) & $37(62.7)$ & $84(60.4)$ & .874 \\
\hline Diabetes mellitus (No.) (\%) & $14(23.7)$ & $24(17.3)$ & .326 \\
\hline Atrial fibrillation (No.) (\%) & $35(59.3)$ & $50(35.9)$ & .003 \\
\hline \multicolumn{4}{|l|}{ Clinical variables } \\
\hline Baseline NIHSS (median) (IQR) & $15(12-18)$ & $14(10-17)$ & .116 \\
\hline Onset-to-puncture time (median) (IQR) (min) & $334(237-425)$ & $306(213-415)$ & .289 \\
\hline Retrieval attempts (median) (IQR) & $2(1-3)$ & $1(1-3)$ & .039 \\
\hline \multicolumn{4}{|l|}{ Radiologic data } \\
\hline Baseline infarct volume (median) (IQR) (mL) & $59.9(34.7-80.9)$ & $46.3(27.5-80.0)$ & .096 \\
\hline Baseline hypoperfusion volume (median) (IQR) (mL) & $108.9(79.2-153.6)$ & $107.1(68.0-160.0)$ & .639 \\
\hline Recanalization (No.) (\%) & $48(81.4)$ & $123(88.4)$ & .181 \\
\hline 24-Hour hemorrhagic transformation (No.) (\%) & $58(98.3)$ & $54(38.8)$ & $<.001$ \\
\hline 24-Hour parenchymal hemorrhage (No.) (\%) & $45(76.3)$ & $6(4.3)$ & $<.001$ \\
\hline
\end{tabular}

Note:-IQR indicates interquartile range.

bolysis. At present, thrombectomy has been demonstrated as an effective therapy for patients with acute ischemic stroke with large-vessel occlusion. ${ }^{15}$ However, the relationship between the occurrence of parenchymal hemorrhage at 24 hours and hyperdense lesions on NCCT immediately after thrombectomy is still unclear.

Here, we propose a new kind of metallic hyperdensity sign combining maximum Hounsfield units and morphologic features to predict $\mathrm{PH}$ at 24 hours. The aim of our study was to evaluate the value of the newly defined metallic hyperdensity sign following MT for predicting PH at 24 hours in patients with acute ischemic stroke with large-vessel occlusion.

\section{MATERIALS AND METHODS}

\section{Ethics Statement}

Written informed consent was obtained from each patient or an appropriate family member. The human ethics committee of the Second Affiliated Hospital of Zhejiang University approved the protocol of this study. All clinical investigations were conducted according to the principles expressed in the Declaration of Helsinki.

\section{Patient Selection}

We analyzed our prospectively collected data base for consecutive patients with acute ischemic stroke who received mechanical thrombectomy between January 2014 and September 2018, as NCCT examination immediately after mechanical thrombectomy was performed since January 2014. We enrolled patients with the following qualifications: 1) They underwent MT due to large-vessel occlusion of the anterior circulation within 24 hours post-symptom onset based on multimodel imaging, 2) they underwent postinterventional
NCCT immediately after MT, and 3) they had a follow-up CT or MR imaging at 24 hours.

\section{Definition of the Metallic Hyperdensity Sign}

All CT examinations were performed on a multidetector CT scanner with axial 5-mm section thickness. "Hyperdense lesion" was defined as the presence of high density within the brain parenchymal area. First, the hyperdense lesions on each layer were manually segmented, respectively, by 2 experienced neurologists (C.X. and Y.Z.), with the open-source software RadiAnt DICOM Viewer (https://www.radiantviewer.com). Second, we measured the density value (Hounsfield unit) of the segmented area on each layer and recorded the maximum value. The metallic hyperdensity sign was defined when a maximum CT density of $>90 \mathrm{HU}$ was identified within the nonpetechial intracerebral hyperdense lesion (diameter, $\geq 1 \mathrm{~cm}$ ) in the basal ganglia.

Two experienced neurologists (X.C. and Z.Y.) blinded to clinical data reviewed images for assessing the presence or absence of the metallic hyperdensity sign on NCCT independently, and the inconsistent data were reviewed by another experienced neurologist and settled by consensus discussion afterward.

\section{Evaluation of $\mathrm{HT}$ and $\mathrm{PH}$}

HT (including hemorrhagic infarction and $\mathrm{PH}$ ) was assessed on the basis of imaging characteristics at 24 hours after MT, referring to the European Cooperative Acute Stroke Study II (ECASS II) criterions. ${ }^{16}$ According to previous studies, intracerebral hyperdense lesions that were no longer discernible on the 24hour follow-up CT were defined as contrast enhancement, while lesions persisting on follow-up CT were considered hemorrhagic lesions. ${ }^{17}$ 

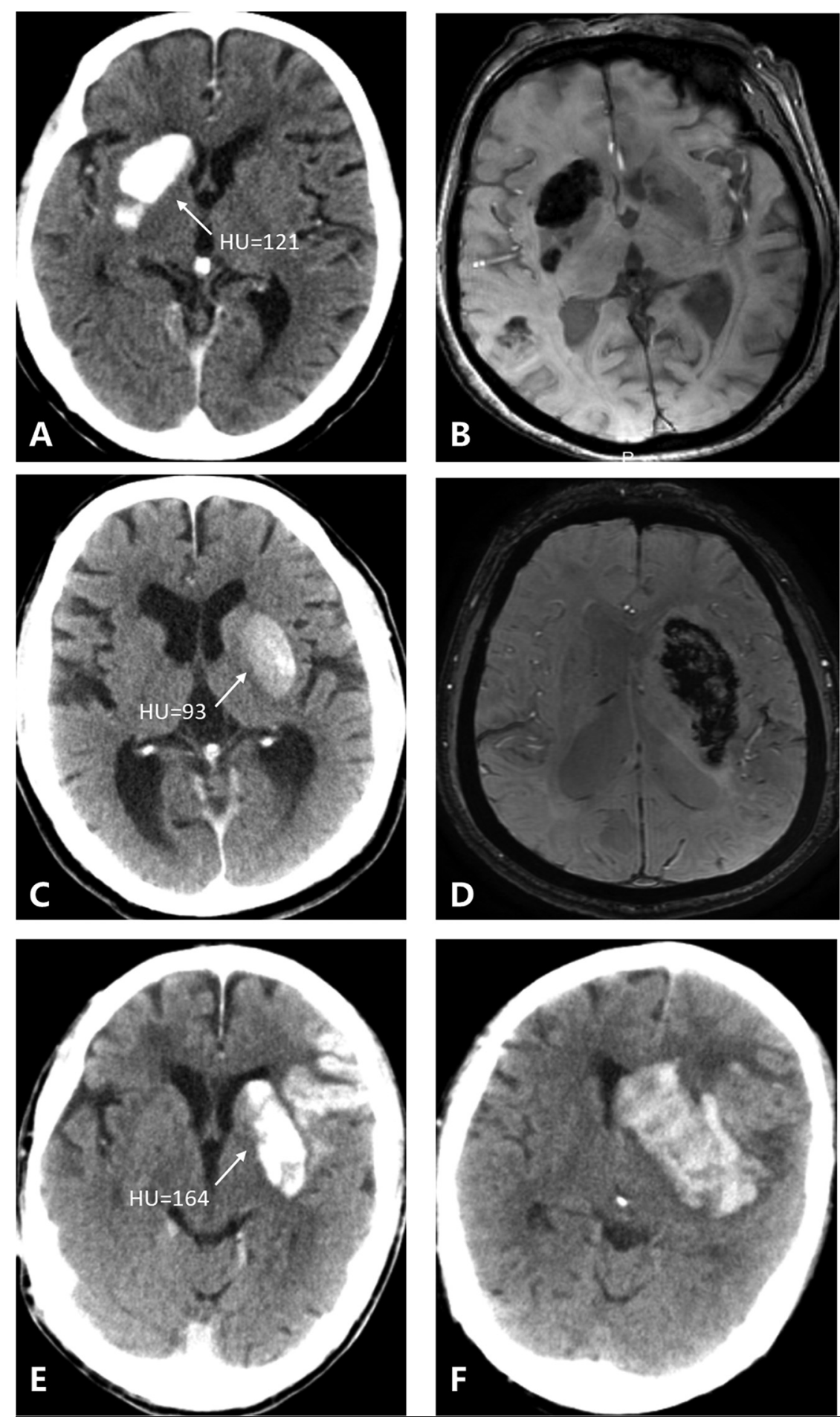

FIG 1. Three patients with 3 kinds of metallic hyperdensity signs. A, C, and $E$, NCCT images obtained immediately after mechanical thrombectomy. $B$ and $D$, SWI at 24 hours after mechanical thrombectomy. F, NCCT image at 24 hours after mechanical thrombectomy. A 77-year-old woman with a hyperdense lesion and a maximum CT density of $121 \mathrm{HU}$ in the right caudate nucleus on NCCT images immediately after mechanical thrombectomy $(A)$ has parenchymal hemorrhage on SWI at 24 hours $(B)$. A 71-year-old man with a hyperdense lesion and a maximum CT density of $93 \mathrm{HU}$ in the left lenticular nucleus on NCCT images immediately after mechanical thrombectomy $(C)$ has parenchymal hemorrhage on SWI at 24 hours $(D)$. An 81-year-old man with a hyperdense lesion and a maximum CT density of $164 \mathrm{HU}$ in the left lenticular nucleus on NCCT images immediately after mechanical thrombectomy $(E)$ has parenchymal hemorrhage on the NCCT image at 24 hours $(F)$.

\section{Statistical Analysis}

All statistical analyses were performed with the SPSS package

(Version 19.0; IBM, Armonk, New York). Metric and normally distributed variables were reported as mean $\pm \mathrm{SD}$; non-normally distributed variables were reported as median and interquartile range. Categoric variables were presented as frequency (percentage). The Fisher exact test was used to compare the dichotomous variables, and the Mann-Whitney $U$ test was used for the non-normally distributed continuous variables. Normally distributed continuous variables were assessed with the Student $t$ test. Statistical significance was set at $P<.05 . \kappa$ statistics were used to test inter- and intrarater reliability for detecting the presence of the metallic hyperdensity sign. Receiver operating characteristic curve analysis was used to determine the predictive value.

\section{RESULTS}

A total of 198 patients were included. The mean age was $69.8 \pm 11.7$ years, and $82(41.4 \%)$ were women. The median baseline NIHSS was 14 (interquartile range $=11-18)$. One hundred twelve (56.5\%) patients had HT, and 51 (25.7\%) patients had $\mathrm{PH}$ at 24 hours.

Intracerebral hyperdense lesions were found in $155(78.3 \%)$ patients, among whom $59(38.1 \%)$ patients had the metallic hyperdensity sign and the remaining 96 (61.9\%) patients had a nonmetallic hyperdensity sign. Moreover, $14(14.6 \%)$ patients had hyperdense lesions in the cortical area; 10 $(10.4 \%)$, in the subcortical area; 64 (66.7\%), in the basal ganglia; and 8 $(8.3 \%)$, in both cortical and subcortical areas and basal ganglia. The inter- and intrarater observer agreement for assessing the presence of intracerebral hyperdense lesions $(\kappa=0.924, \kappa=$ $0.947)$ and the presence of the metallic hyperdensity sign $(\kappa=0.874, \kappa=$ 0.912 ) was good. Patients with the metallic hyperdensity sign were more likely to have $\mathrm{PH}$ than those without the sign $(76.3 \%$ versus $4.3 \%$; odds ratio, 71.250; odds ratio, 75.553 after adjusting for atrial fibrillation, onset to puncture time, and recanalization; $P<.001)$. The sensitivity, specificity, positive predictive value, and negative predictive value of the metallic hyperdensity sign in predicting $\mathrm{PH}$ were $88.2 \%$ (95\% CI, 79.1\%-97.4\%), 90.5\% (95\% CI, 85.7\%-95.3\%), 76.3\% (95\% CI, 65.1\%-87.4\%), and 95.7\% (95\% CI, 92.3\%-99.1\%), respectively (Table 1). 

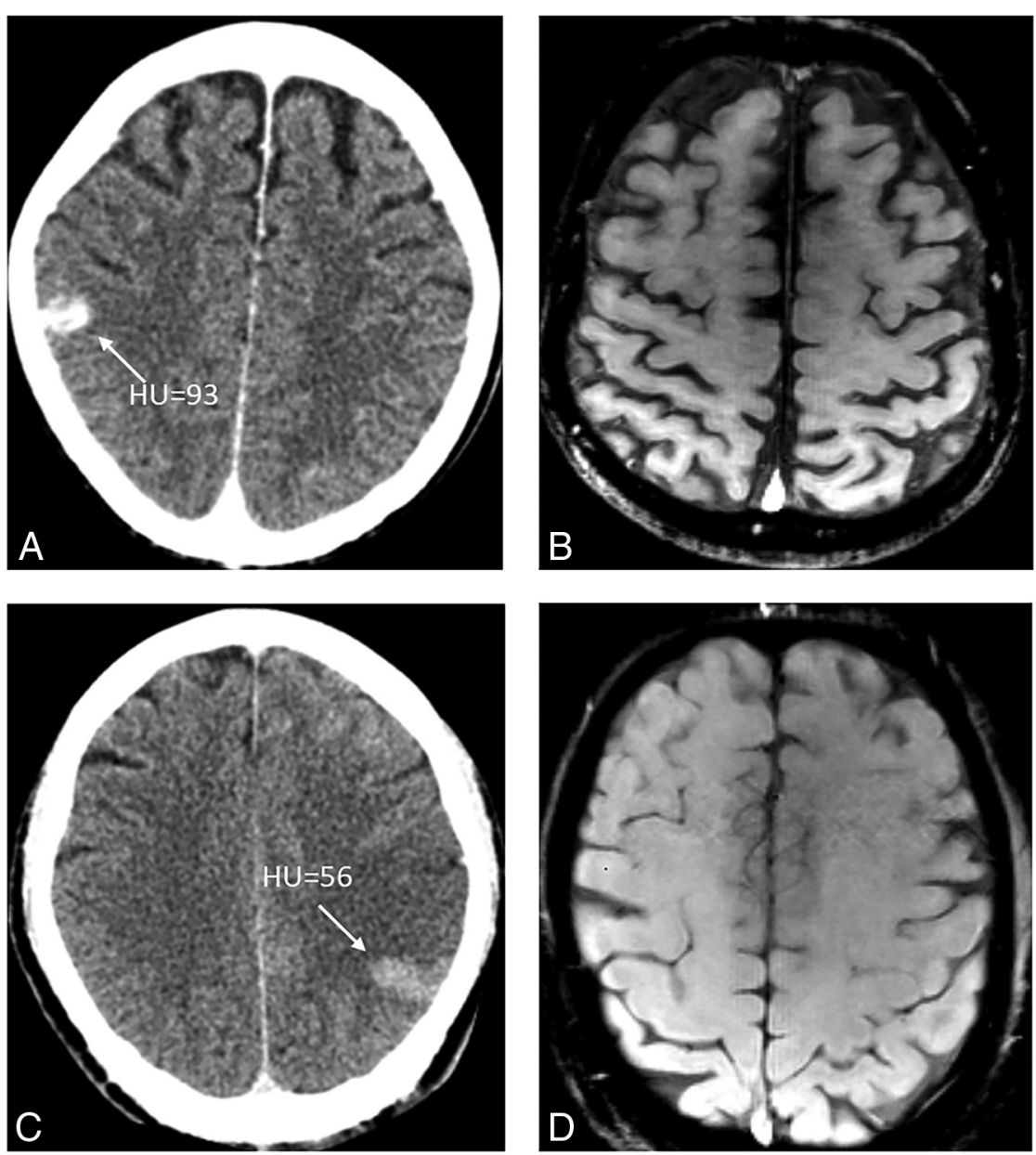

FIG 2. Two patients who show the typical cortical hyperdense lesion on NCCT immediately after MT ( $A$ and $C$ ) have no HT on follow-up SWI ( $B$ and $D)$.

Patients with the metallic hyperdensity sign were more likely to have atrial fibrillation $(P=.003)$ and underwent more retrieval attempts $(P=.039)$ than those without the sign. Other demographic and clinical variables including age, sex, hypertension, diabetes mellitus, baseline NIHSS, baseline hypoperfusion volume, onset-to-puncture time, and recanalization did not significantly differ between patients with and without the metallic hyperdensity sign (Table 2).

Figure 1 shows 3 patients with 3 kinds of metallic hyperdensity signs.

\section{DISCUSSION}

Our study demonstrates that the metallic hyperdensity sign on NCCT performed immediately after MT can predict $\mathrm{PH}$ at 24 hours with high specificity and negative predictive value.

Intracerebral hyperdense lesions were first reported on NCCT immediately after intra-arterial thrombolysis in patients with acute ischemic stroke early in the 1990s. ${ }^{18,19}$ The rates of intracerebral hyperdense lesions shown on NCCT after intra-arterial reperfusion therapy varied greatly, from $32.9 \%$ to $84.2 \%{ }^{13,14,17,20}$ The reason for such a wide variation may be due to the differences in the definition of hyperdense lesions, number of patients, recanalization rate, and the quality of the imaging equipment. ${ }^{21} \mathrm{Com}-$ pared with previous studies, the incidence of intracerebral hyper- dense lesions in our center was relatively high (our center versus other centers, $83.3 \%$ versus $32.9 \%-84.2 \%$ ); this finding may result from the relatively high recanalization rate (our center versus other centers, $86.3 \%$ versus $77.2 \%$ $80 \%)^{21,22}$ In addition, MT would increase the direct damage to the endothelium of cerebral arteries when pulling thrombus, ${ }^{23}$ compared with intra-arterial thrombolysis, which might increase the incidence of intracerebral hyperdense lesions.

Currently, the most widely accepted hypothesis is that intracerebral hyperdense lesions are caused by leakage of contrast medium into the extracellular spaces via vessels, as a result of microvascular damage and increased permeability of the BBB. ${ }^{17,21,22}$ Yoon et al ${ }^{13}$ documented that high-density areas $(>90$ HU) found on NCCT after intra-arterial thrombolysis are closely associated with $\mathrm{PH}$, possibly due to pathologic changes of ischemic injury to the degradation of the basal lamina, a structural barrier. Moreover, $\mathrm{PH}$ is the result of severe microvascular damage extended to the basal lamina. In our study, 51 (25.7\%) patients had $\mathrm{PH}$ at 24 hours, which is higher than that in published trials such as the Multicenter Randomized Clinical trial of Endovascular treatment for Acute ischemic stroke in the Netherlands (MR CLEAN), Endovascular Treatment for Small Core and Proximal Occlusion Ischemic Stroke (ESCAPE), Extending the Time for Thrombolysis in Emergency Neurological Deficits-Intra-Arterial (EXTENDIA), Solitaire with the Intention for Thrombectomy as Primary Endovascular Treatment (SWIFT PRIME), and Endovascular Revascularization With Solitaire Device Versus Best Medical Therapy in Anterior Circulation Stroke Within 8 Hours (REVASCAT) at $6 \%, 3.6 \%, 11 \%, 5 \%$, and $5.8 \%$, respectively. ${ }^{1-5}$ However, our rate is actually based on the real-world data for mechanical thrombectomy. The median time from stroke onset to groin puncture in these above studies was not $>270$ minutes (4.5 hours), which, to some extent, is difficult to achieve in the real world. Indeed, in previous retrospective studies, the occurrence of $\mathrm{PH}$ varied greatly, from $5.9 \%$ to $23.5 \% .{ }^{17,24}$ Another reason for the relatively higher rate of $\mathrm{PH}$ is the use of SWI to detect hemorrhage because SWI accounted for $48 \%$ of the 24 -hour follow-up examinations, which may result in a visual overestimation in hematoma size due to susceptibility effects. ${ }^{25}$

In addition, we found that the metallic hyperdensity sign was more likely in patients with atrial fibrillation and a higher number of retrieval attempts. Actually, the extent of BBB disruption was more severe in cardiogenic embolism stroke than in other stroke 

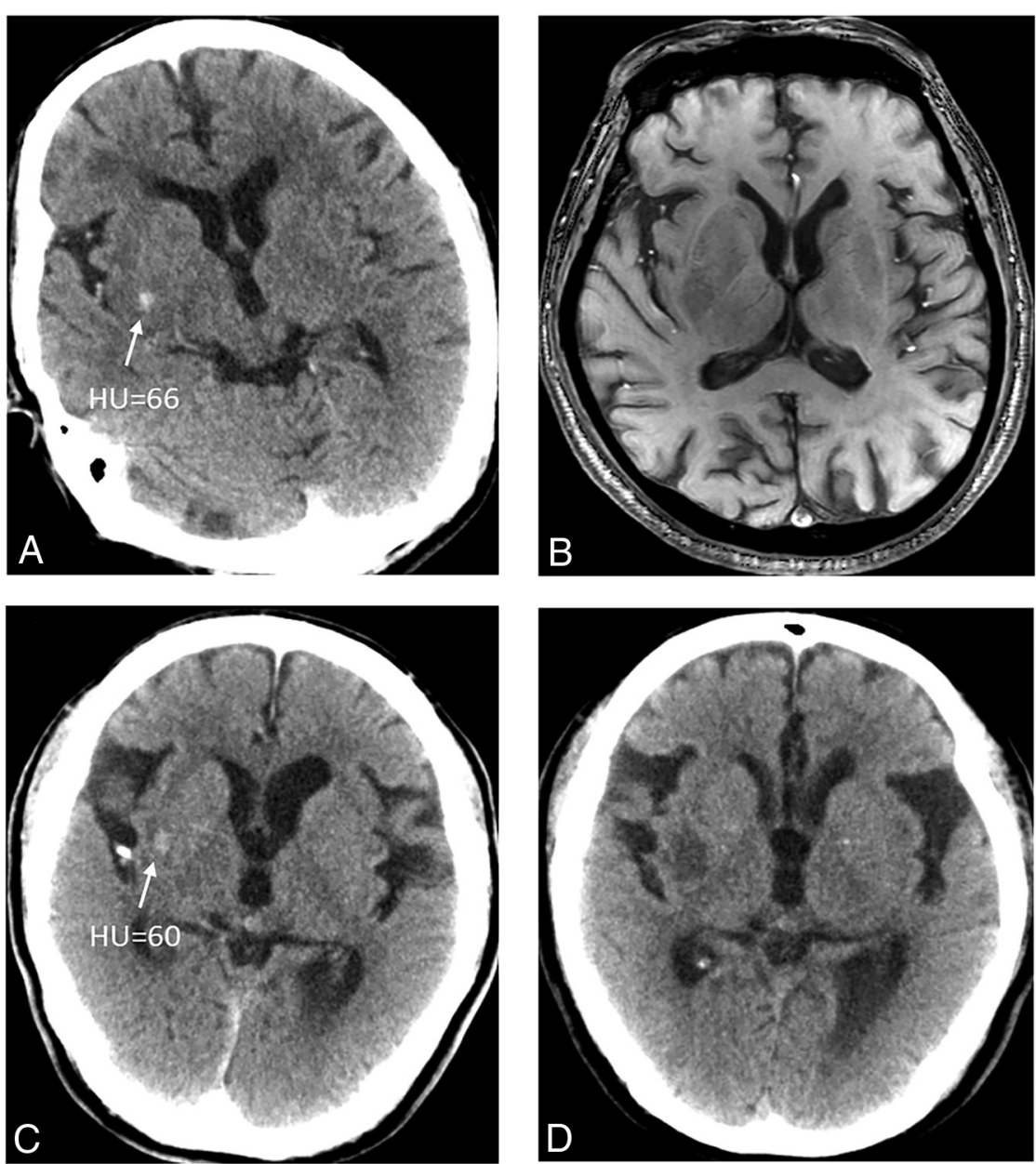

FIG 3. Two patients who show a petechial hyperdense lesion in the basal ganglia on NCCT immediately after MT ( $A$ and $C$ ) have no HT on follow-up SWI $(B)$ and the remaining low densities on follow-up NCCT (D).
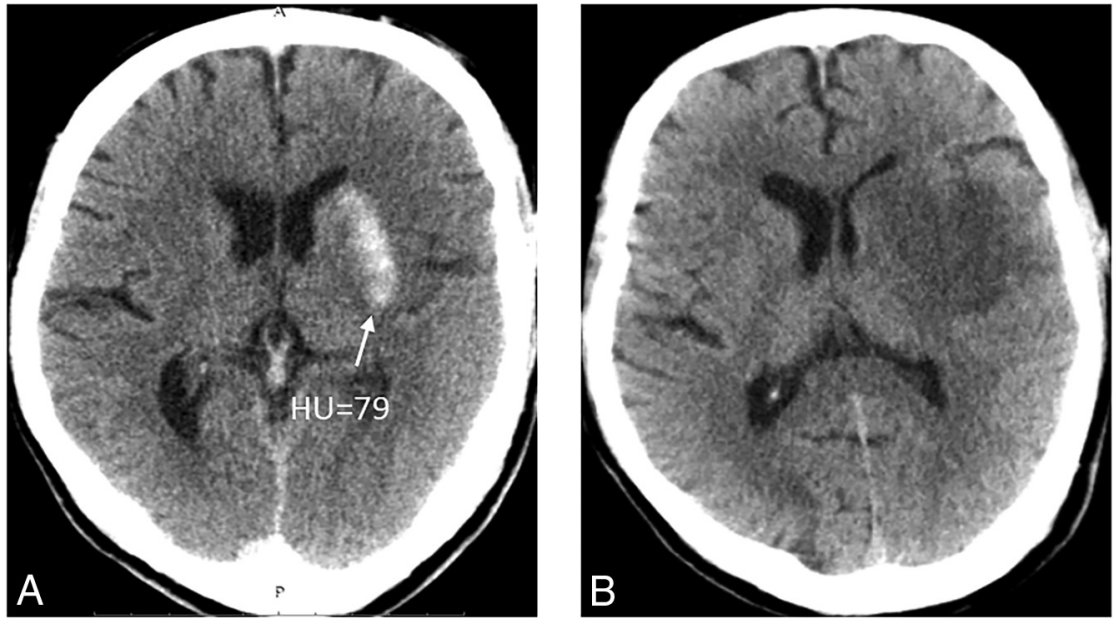

FIG 4. A patient who shows a nonpetechial hyperdense lesion on NCCT immediately after MT (A) has no HT on follow-up NCCT and the remaining low densities (B).

subtypes during the hyperacute stage ${ }^{26}$; with the increased number of retrieval attempts, the damage to the endothelium of cerebral arteries would be more severe. ${ }^{23}$ Besides, previous experimental research has shown that massive HT, with the distinguishing features of a rapid extravasation of blood leading to the compression of contiguous tissues, occurs when the BBB integrity is lost. ${ }^{27}$ Therefore, we propose that the presence of our defined metallic hyperdensity sign in the current study might reflect severe microvascular damage and extensive $\mathrm{BBB}$ disruption because the study focused on those subjects with relatively high contrast leakage, which leads to massive HT, especially $\mathrm{PH}$.

To our knowledge, this is the first study focusing on both the density and extent of intracerebral hyperdense lesions on NCCT performed immediately after MT and its predictive value for $\mathrm{PH}$ at 24 hours. The metallic hyperdensity sign, a readily available and reliable NCCT-based imaging marker, might be helpful in identifying those patients with a higher risk of $\mathrm{PH}$ after MT and is easily applied in clinical practice. It may not only provide prognostic information for clinicians and patients but also be helpful in clinical management after MT. For instance, patients with the metallic hyperdensity sign should receive all possible care, including relatively strict blood pressure control and delayed or no antiplatelet and anticoagulation treatments.

Limitations included the retrospective nature in our single stroke center and the moderate number of cases, resulting in a potential risk of selection bias, though data were prospectively established using a stroke registry. Second, there was heterogeneity in the evaluation of $\mathrm{PH}$, which was due to 2 methods of follow-up imaging (NCCT versus SWI, 52\% versus $48 \%$ ). Third, despite our effort to find the optimal Hounsfield unit threshold for the metallic hyperdensity sign, the selection of $90 \mathrm{HU}$ was still based on previous literature. In addition, 6 patients in our study without the metallic hyperdensity sign on NCCT obtained immediately after MT still developed $\mathrm{PH}$ on follow-up images. Although the number of these patients is very small, the causes and mechanisms of this situation and the optimal HU threshold are worthy of further study. Although a CT scan obtained at least 19-24 hours after endovascular therapy was reported as a reliable method to differentiate contrast from hemorrhage, ${ }^{28}$ contrast enhancement could still be relatively dense 24 hours after thrombectomy, and the differentiation would be better with the extended time after an intervention. Finally, we did not measure BBB per- 

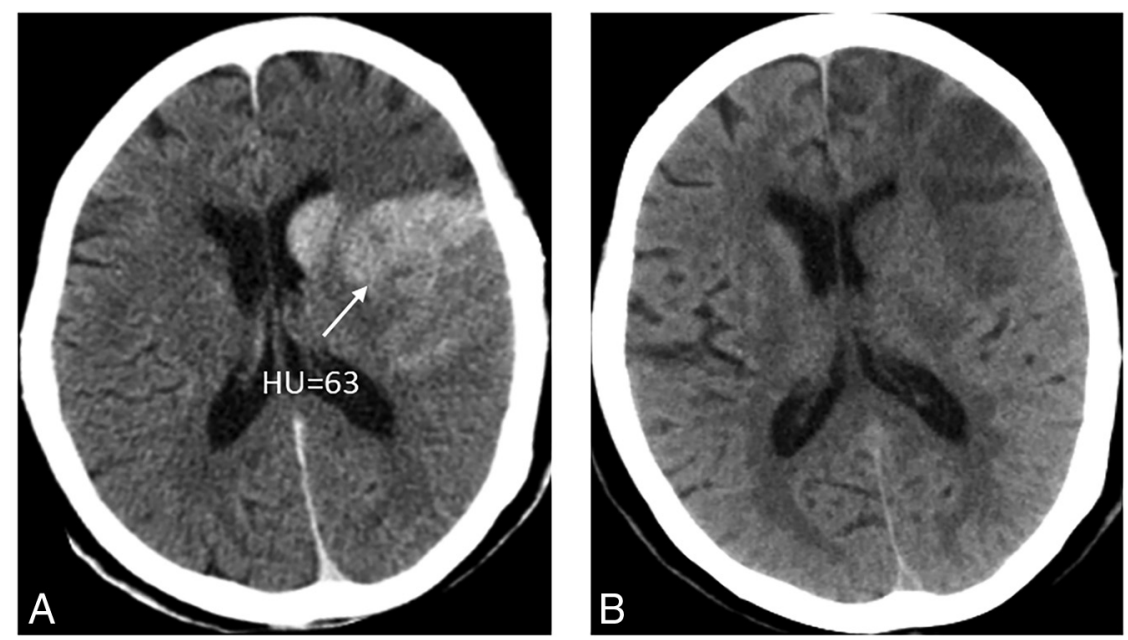

FIG 5. A patient who shows the subcortical hyperdense lesion involving both the basal ganglia and subcortical white matter on NCCT immediately after MT (A) has no HT on NCCT immediately after MT and the remaining low densities on follow-up NCCT (B).
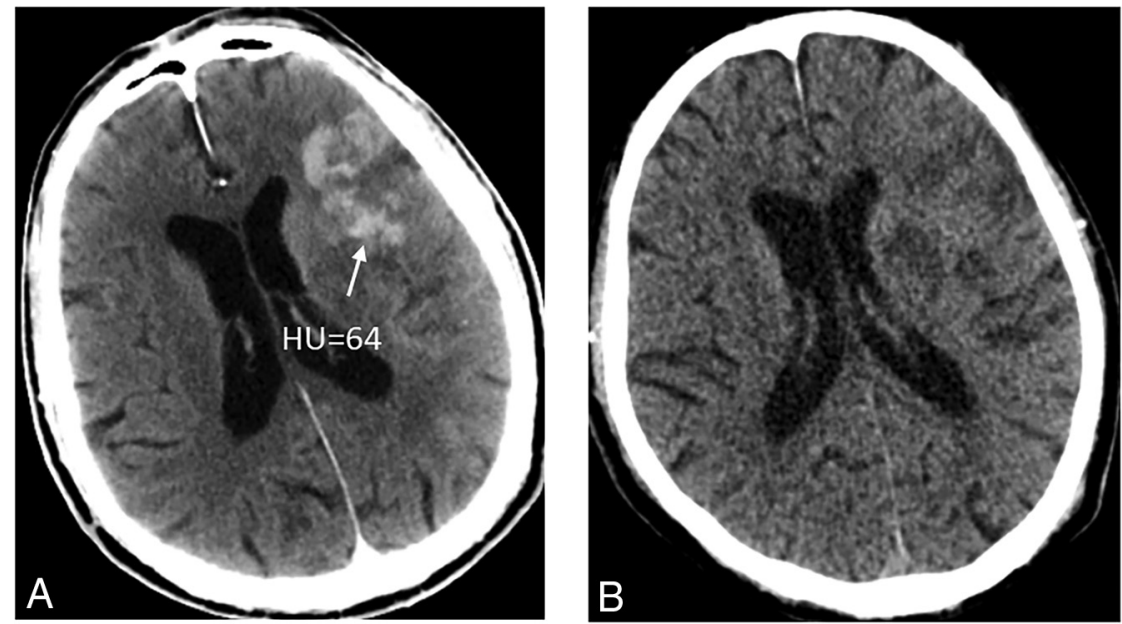

FIG 6. A patient who shows the subcortical hyperdense lesion on NCCT immediately after MT $(A)$ but has no HT on follow-up NCCT and the remaining low densities $(B)$.

meability on multimodal imaging, and the underlying mechanisms of hyperdense intracerebral lesions need to be clarified with further experimental and pathologic studies.

\section{CONCLUSIONS}

The NCCT-based metallic hyperdensity sign immediately after MT in patients with large-vessel occlusion may indicate a high risk of $\mathrm{PH}$ at 24 hours with a high specificity of $90.5 \%$ (odds ratio, 90.576; 95\% CI, 85.7\%-95.3\%) and negative predictive value of 95.7\% (odds ratio, 95.683; 95\% CI, 92.3\%-99.1\%), which may be helpful in postinterventional management 24 hours after mechanical thrombectomy.

Disclosures: Min Lou—RELATED: Grant: National Natural Science Foundation of China (81471170 and 81622017), Fundamental Research Funds for the Central Universities (2017XZZX002-09), and the National Key Research and Development Program of China (2016YFC1301503).* *Money paid to the institution.

\section{REFERENCES}

1. Berkhemer OA, Fransen PS, Beumer D, et al. A randomized trial of intraarterial treatment for acute ischemic stroke. NEnglJMed 2015; 372:11-20 CrossRef Medline
2. Campbell BC, Mitchell PJ, Kleinig TJ, et al; EXTEND-IA Investigators. Endovascular therapy for ischemic stroke with perfusion-imaging selection. N Engl J Med 2015;372:1009-18 CrossRef Medline

3. Goyal M, Demchuk AM, Menon BK, et al; ESCAPE Trial Investigators. Randomized assessment of rapid endovascular treatment of ischemic stroke. N Engl J Med 2015;372:1019-30 CrossRef Medline

4. Jovin TG, Chamorro A, Cobo E, et al; REVASCAT Trial Investigators. Thrombectomy within 8 hours after symptom onset in ischemic stroke. N Engl J Med 2015;372:2296-306 CrossRef Medline

5. Saver JL, Goyal M, Bonafe A, et al; SWIFT PRIME Investigators. Stent-retriever thrombectomy after intravenous T-PA vs. t-PA alone in stroke. $N$ Engl J Med 2015;372:2285-95 CrossRef Medline

6. Volny O, Krajina A, Belaskova S, et al. Mechanical thrombectomy performs similarly in real world practice: a 2016 nationwide study from the Czech Republic. J Neurointerv Surg 2018;10:741-45 CrossRef Medline

7. Serles W, Gattringer T, Mutzenbach S, et al; Austrian Stroke Unit Registry Collaborators. Endovascular stroke therapy in Austria: a nationwide 1-year experience. Eur J Neurol 2016;23:906-11 CrossRef Medline

8. Jiang S, Fei A, Peng Y, et al. Predictors of outcome and hemorrhage in patients undergoing endovascular therapy with Solitaire stent for acute ischemic stroke. PLoS One 2015;10:e0144452 CrossRef Medline 
9. van Kranendonk KR, Treurniet KM, Boers AMM, et al; MR CLEAN investigators. Hemorrhagic transformation is associated with poor functional outcome in patients with acute ischemic stroke due to a large vessel occlusion. J Neurointerv Surg 2018 Oct 8. [Epub ahead of print] CrossRef Medline

10. Kellert L, Hametner C, Rohde S, et al. Endovascular stroke therapy: tirofiban is associated with risk of fatal intracerebral hemorrhage and poor outcome. Stroke 2013;44:1453-55 CrossRef Medline

11. Payabvash S, Khan AA, Qureshi MH, et al. Detection of intraparenchymal hemorrhage after endovascular therapy in patients with acute ischemic stroke using immediate postprocedural flat-panel computed tomography scan. J Neuroimaging 2016;26:213-18 CrossRef Medline

12. Payabvash S, Qureshi MH, Khan SM, et al. Differentiating intraparenchymal hemorrhage from contrast extravasation on postprocedural noncontrast CT scan in acute ischemic stroke patients undergoing endovascular treatment. Neuroradiology 2014; 56:737-44 CrossRef Medline

13. Yoon W, Seo JJ, Kim JK, et al. Contrast enhancement and contrast extravasation on computed tomography after intra-arterial thrombolysis in patients with acute ischemic stroke. Stroke 2004;35: 876-81 CrossRef Medline

14. Jang YM, Lee DH, Kim HS, et al. The fate of high-density lesions on the non-contrast CT obtained immediately after intra-arterial thrombolysis in ischemic stroke patients. Korean J Radiol 2006;7: 221-28 CrossRef Medline

15. Powers WJ, Rabinstein AA, Ackerson T, et al; American Heart Association Stroke Council. 2018 Guidelines for the Early Management of Patients with Acute Ischemic Stroke: A Guideline for Healthcare Professionals From the American Heart Association/ American Stroke Association. Stroke 2018;49:e46-110 CrossRef Medline

16. Larrue V, von Kummer RR, Müller A, et al. Risk factors for severe hemorrhagic transformation in ischemic stroke patients treated with recombinant tissue plasminogen activator: a secondary analysis of the European-Australasian Acute Stroke Study (ECASS II). Stroke 2001;32:438-41 CrossRef Medline

17. Lummel N, Schulte-Altedorneburg G, Bernau C, et al. Hyperattenuated intracerebral lesions after mechanical recanalization in acute stroke. AJNR Am J Neuroradiol 2014;35:345-51 CrossRef Medline

18. Komiyama M, Nishijima Y, Nishio A, et al. Extravasation of contrast medium from the lenticulostriate artery following local intracarotid fibrinolysis. Surg Neurol 1993;39:315-19 CrossRef Medline

19. Wildenhain SL, Jungreis CA, Barr J, et al. CT after intracranial intraarterial thrombolysis for acute stroke. AJNR Am J Neuroradiol 1994;15:487-92 Medline

20. Nakano S, Iseda T, Kawano H, et al. Parenchymal hyperdensity on computed tomography after intra-arterial reperfusion therapy for acute middle cerebral artery occlusion: incidence and clinical significance. Stroke 2001;32:2042-48 CrossRef Medline

21. Parrilla G, García-Villalba B, Espinosa de Rueda M, et al. Hemorrhage/contrast staining areas after mechanical intra-arterial thrombectomy in acute ischemic stroke: imaging findings and clinical significance. AJNR Am J Neuroradiol 2012;33:1791-96 CrossRef Medline

22. Tomsick T. Hyperattenuated intracerebral lesions after mechanical recanalization in acute stroke: contrast and compare. AJNR Am J Neuroradiol 2014;35:352-53 CrossRef Medline

23. Teng D, Pannell JS, Rennert RC, et al. Endothelial trauma from mechanical thrombectomy in acute stroke: in vitro live-cell platform with animal validation. Stroke 2015;46:1099-106 CrossRef Medline

24. Kim JT, Heo SH, Cho BH, et al. Hyperdensity on non-contrast CT immediately after intra-arterial revascularization. J Neurol 2012; 259:936-43 CrossRef Medline

25. Burgess RE, Warach S, Schaewe TJ, et al. Development and validation of a simple conversion model for comparison of intracerebral hemorrhage volumes measured on CT and gradient recalled echo MRI. Stroke 2008;39:2017-20 CrossRef Medline

26. Liu C, Shi F, Chen Z, et al. Severe blood-brain barrier disruption in cardioembolic stroke. Front Neurol 2018;9:55 CrossRef Medline

27. Jickling GC, Liu D, Stamova B, et al. Hemorrhagic transformation after ischemic stroke in animals and humans. J Cereb Blood Flow Metab 2014;34:185-99 CrossRef Medline

28. Dekeyzer S, Nikoubashman O, Lutin B, et al. Distinction between contrast staining and hemorrhage after endovascular stroke treatment: one CT is not enough. J Neurointerv Surg 2017;9:394-98 CrossRef Medline 